\title{
Near-Optimal Zero Correlation Zone Sequence Sets from Paraunitary Matrices
}

\author{
Shibsankar Das*, Udaya Parampalli ${ }^{\star}$, Sudhan Majhi ${ }^{\dagger}$, and Zilong Liu ${ }^{\ddagger}$ \\ ${ }^{*}$ Department of Mathematics, Indian Institute of Technology Patna, Bihar, India \\ ${ }^{\star}$ School of Computing and Information Systems, University of Melbourne, Victoria, Australia \\ ${ }^{\dagger}$ Department of Electrical Engineering, Indian Institute of Technology Patna, Bihar, India \\ ${ }^{\ddagger}$ Institute for Communication Systems, 5G Innovation Centre, University of Surrey, U.K. \\ Email:\{shibsankar.pma15\}@iitp.ac.in
}

\begin{abstract}
Zero correlation zone (ZCZ) sequence sets play an important role in interference-free quasi-synchronous code-division multiple access communications. In this paper, for the first time, we investigate the periodic correlation properties of polyphase sequences obtained from paraunitary (PU) matrices, which shows the inherent relationship between PU matrix and ZCZ sequence sets. Our investigation suggests that any arbitrary PU matrix can produce $\mathrm{ZCZ}$ sequence sets by controlling its expanded form. The key idea is to impose certain restrictions on the expanded forms of the PU matrices to enable precise computation of the periodic correlation functions of the constructed sequences. We show that our proposed construction leads to near-optimal ZCZ sequence sets with regard to the ZCZ set size upper bound.
\end{abstract}

\section{INTRODUCTION}

Code-division multiple-access (CDMA) has been an important multiuser communication scheme based on signature sequences. Among many CDMA proposals, quasisynchronous CDMA (QS-CDMA) is interesting as it helps to simply the system design and offers enhanced error rate performance [1]. In a QS-CDMA system, the relative time delay between the signals of different users is random but limited to a certain time range $T$, where $T$ is smaller than the sequence length. The spreading sequences characterize the associated multiple-access interference and multi-path interference properties in the system [2]. In a QS-CDMA system, it is desirable that the spreading sequences can have zero correlation zone (ZCZ) within the time delay $T$ for the elimination of both multiple-access interference and multi-path interference. In addition, $\mathrm{ZCZ}$ sequence sets have also been used in channel estimation [3], radar [4], pilot design [5], and so on.

From the definition, ZCZ sequences possess zero periodic auto-correlation function (PACF) and periodic crosscorrelation function (PCCF) over a region around the origin. That is, the PACF and PCCF of the sequences are zero within the range $|\tau| \leq T$, where $\tau$ refers to timeshift variable and $T$ is the width of ZCZ. In this paper, the time window spanning from $-T$ to $T$ is referred to as the interference free window of $\mathrm{ZCZ}$ sequence set. In [6], Tang, Fan and Matsufuji have derived the theoretical upper bound $T \leq L / M-1$, where $L$ is the sequence length and $M$ refers to the number of sequences in the set. To support large number of users, it is preferable that $M$ should be as large as possible. According to the theoretical bound on $T$, the performance parameter $\eta$, defined by $\eta=(T+1) M / L[7]$, can be used to evaluate the efficiency of the construction method. So far, an interesting research direction has been to construct ZCZ sequence sets with performance parameter greater than $1 / 2$.

In the literature, ZCZ sequence sets have been constructed based on Boolean functions [8], [9], perfect sequences [10], [11], mismatched filtering [12], and so on. An important research direction is to generate $\mathrm{ZCZ}$ sequence sets based on complementary sets of sequences (CSSs) [13], [14]. By definition, a set of sequences is said to be a CSS if the sum of aperiodic auto-correlation functions is zero for any non-zero time-shift. Two distinct CSSs are called mutually orthogonal CSSs (MOCSSs) if the sum of aperiodic cross-correlation functions between them is zero for any time-shift. The concept of MOCSSs was introduced by Tseng and Liu [15]. Recursive and direct (Boolean) algorithms for generating CSSs were proposed by Budišin [16] and Davis [17], respectively. MOCSSs with maximum number of CSSs are also called complete complementary codes (CCC) [18].

In [19], a compact formulation described in $Z$-domain framework has been proposed for complementary sequence pairs (and CSSs) by using paraunitary (PU) matrices. The concept of PU matrices was introduced by Vaidyanathan in the theory of filter banks [20]. A PU matrix is a matrix of polynomials in the indeterminate variable $Z^{-1}$ which is unitary on the unit circle, i.e., $|Z|=1$. Recently, the applications of PU matrices have been extended to $q$-ary CSSs [21] and QAM CSSs [22]. [21] introduced the use of Butson-type Hadamard $(B H)$ matrices to produce $q$-ary sequences. In [23], it has been shown that the use of equivalent forms of $B H$ matrices can significantly increase the number of generated sequences in the PU matrix approach. It is worth mentioning that all sequences constructed by the direct (Boolean) approach have length $2^{n}$, while the PU matrix approach can generate sequences with length non-power of two as well. In addition, the $Z$-domain framework greatly simplifies the derivations of the construction methods. Another important advantage of PU matrices is the energy balance property which can avoid the noise amplification problem [20]. In recent years, there has been a surge of research interest on PU matrices in the construction of CCCs [23]-[27]. In [24, Th.1], it is shown that there exists an equivalence between a square PU matrix and CCC, when the latter is expressed with polynomial entries. That is, each column is a CSS and two distinct columns are 
MOCSSs. Since CCC can be efficiently generated by PU matrices, and $\mathrm{CCC}$ can lead to ZCZ sequence sets, we ask the following question:

"How can we directly construct ZCZ sequence sets from PU matrices?"

In this paper, we extend the application of PU matrices to the construction of $\mathrm{ZCZ}$ sequence sets with performance parameter greater than $1 / 2$. Specifically, we draw out the inherent connections between $\mathrm{ZCZ}$ sequences and PU matrix. Our proposed method can produce a large class of ZCZ sequences with more flexible parameters (e.g., sequence lengths other than power of two) by using the equivalent forms of $\mathrm{BH}$ matrices. The key idea of our proposed construction is the intrinsic periodic properties of the row sequences of a specific unitary matrix for the expanded form of PU matrix. We show that our proposed construction can generate near-optimal ZCZ sequence sets.

The rest of the paper is organized as follows. In Section II, we give some preliminary definitions and notations. In Section III, we propose a simple construction of ZCZ sequence sets based on PU matrices. Finally, we conclude our work in Section IV.

\section{PRELIMINARIES}

We will extensively use the following notations throughout this paper.

1) The $Z$-transform of aperiodic cross-correlation function (ACCF) $R_{x, y}[\tau]$ between two length- $L$ complex-valued sequences $\boldsymbol{x}$ and $\boldsymbol{y}$ is denoted by $R_{x, y}(Z)=\sum_{\tau=0}^{L-1} R_{x, y}[\tau] \cdot Z^{-\tau}=x\left(Z^{-1}\right) \cdot y^{*}(Z)$, where $(\cdot)^{*}$ denotes complex conjugate (see [23]).

2) For a matrix $\mathbf{X}(Z)$ of polynomials over $Z^{-1}$, the tilde operator is defined by $\widetilde{\mathbf{X}(Z)}=\mathbf{X}^{H}\left(Z^{-1}\right)$, where $H$ is the Hermitian operation.

3) A diagonal matrix is denoted by ' $\operatorname{diag}(\cdot)$ '.

4) Butson-type Hadamard matrix $B H(M, Q)$ refers to a complex Hadamard matrix of size $M \times M$ with $Q^{\text {th }}$ roots of unity entries [28]. $B H(M, 2)$ represents a Hadamard matrix denoted by $\mathbf{H}_{M}$ for $M=2,4 m$ and $B H(M, M)$ represents discrete Fourier transform (DFT) matrix denoted by $\mathbf{F}_{M}$.

\section{$A, \mathrm{PCCF}$ and PACF}

Given two length- $L$ complex-valued sequences $\boldsymbol{x}$ and $\boldsymbol{y}$, the periodic correlation function between $\boldsymbol{x}$ and $\boldsymbol{y}$ is defined by

$$
\hat{R}_{x, y}[\tau]=\sum_{k=0}^{L-1} x[k] \cdot y^{*}\left[(k+\tau)_{L}\right], \quad 0 \leqslant \tau \leqslant L-1
$$

where $(k+\tau)_{L}$ is understood to be modulo $L$.

The function $\hat{R}_{x, y}[\tau]$ is called the periodic crosscorrelation function (PCCF) when $\boldsymbol{x} \neq \boldsymbol{y}$; otherwise, it is called periodic auto-correlation function (PACF). For simplicity, the PACF of $\boldsymbol{x}$ will be denoted by $\hat{R}_{\boldsymbol{x}}[\tau]$. Since $\hat{R}_{x, y}[-\tau]=\hat{R}_{\boldsymbol{y}, \boldsymbol{x}}^{*}[\tau]$, it is sufficient to calculate the PCCF $\hat{R}_{x, y}[\tau]$ only for $0 \leq \tau \leq L-1$. For DFT matrix $\mathbf{F}_{M}$, the PCCF between any two rows $\boldsymbol{f}_{m}$ and $\boldsymbol{f}_{m^{\prime}}$ of $\mathbf{F}_{M}$ is given by

$$
\hat{R}_{f_{m}, f_{m^{\prime}}}[\tau]=\omega^{-m \tau} \delta\left(m-m^{\prime}\right)
$$

That is, the PCCF of two different rows of DFT matrix is zero for all shifts. These rows are referred to as uncorrelated sequences. Note that DFT matrices form a subclass of $B H$ matrices.

\section{B. ZCZ Sequence Sets}

Let $\mathbf{x}(Z)=\left[x^{(0)}(Z), x^{(1)}(Z), \cdots, x^{(M-1)}(Z)\right]^{T}$ be a set of $M$ sequences, each of length $L$. The zero periodic auto-correlation zone $T_{A C Z}$ and the zero periodic cross-correlation zone $T_{C C Z}$ of this sequence set $\mathbf{x}(Z)$ are defined to be

$T_{A C Z}=\max \left\{T: \hat{R}_{\boldsymbol{x}^{(m)}}[\tau]=0, \forall m,|\tau| \leq T, \tau \neq 0\right\}$

$T_{C C Z}=\max \left\{T: \hat{R}_{\boldsymbol{x}^{(m)}, \boldsymbol{x}^{\left(m^{\prime}\right)}}[\tau]=0, \forall m \neq m^{\prime},|\tau| \leq T\right\}$.

Then, the interference free window (IFW) of the sequence set $\mathbf{x}(Z)$ is defined by $T=\min \left\{T_{A C Z}, T_{C C Z}\right\}$.

Definition 1: The set $\mathbf{x}(Z)$ with IFW of $T$ is said to constitute a ZCZ sequence set of $M$ sequences of length $L$ and is denoted by $(M, L, T)-Z C Z$.

A set of sequences is called ZCZ-CSS if it is both CSS and $\mathrm{ZCZ}$ sequence set. The performance parameter is defined by $\eta=(T+1) M / L$, which can be used to evaluate the efficiency of the construction method.

\section{Paraunitary (PU) Matrices}

A PU matrix refers to a matrix of polynomials over $Z^{-1}$ which is unitary on the unit circle, i.e., $|Z|=1$ [20]. That is, PU matrix is a generalization of unitary matrix. A matrix $\mathbf{X}(Z)$ of polynomials with size $M \times K$ is defined as $\mathbf{X}(Z)=\left[x_{l m}(Z)\right]$, where $x_{l m}(Z)$ is a polynomial over $Z^{-1}$ and $0 \leqslant l \leqslant M-1,0 \leqslant m \leqslant K-1$.

Definition 2 (see [20]): A polynomial matrix $\mathbf{X}(Z)$ of size $M \times K$ is said to be a wide sense PU matrix if the following equation is satisfied:

$$
\widetilde{\mathbf{X}(Z)} \cdot \mathbf{X}(Z)=c \cdot \mathbf{I}_{K},
$$

where $c$ is a real positive constant, $\mathbf{I}_{K}$ is an identity matrix of size $K \times K$.

According to [20], any arbitrary PU matrix can be factorized into a product of unitary and diagonal matrices. This factorization is said to be an expanded form of PU matrix. The degree of a PU matrix refers to the minimum number of delays required to implement it. The length of a PU matrix refers to the length of the constituent sequences. A PU matrix is called a unimodular PU matrix if it has only unimodular coefficients. For example, a PU matrix with \pm 1 coefficients refers to a binary PU matrix.

Based on the definitions of CCC and PU matrices, we state the previous result on PU matrices.

Result 1 (see [24]): The matrix $\mathbf{X}(Z)$ represents a polyphase $(M, M, L)$-CCC if and only if it is an $M \times M$ unimodular PU matrix of degree $L-1$.

Example 1: Let $M=K=2$. Then, a $2 \times 2$ binary PU matrix $\mathbf{X}(Z)$ with sequence length 4 is given by

$\mathbf{X}(Z)=\left[\begin{array}{cc}1+Z^{-1}+Z^{-2}-Z^{-3} & 1+Z^{-1}-Z^{-2}+Z^{-3} \\ 1-Z^{-1}+Z^{-2}+Z^{-3} & 1-Z^{-1}-Z^{-2}-Z^{-3}\end{array}\right]$.

It is easy to verify that $S_{\mathbf{x}_{\mu}, \mathbf{x}_{\nu}}(Z)=c \cdot \delta(\mu-\nu), \mu, \nu=$ 0,1 . Thus, $\mathbf{X}(Z)$ represents a set of $(2,2,4)$-CCC. 


\section{CONSTRUCTION OF ZCZ SEQUENCE SETS BASED ON PARAUNITARY MATRICES}

In this section, we will first state our previous construction of unimodular PU matrices with more flexible sequence lengths. Then, we will propose a new construction of ZCZ sequence sets based on PU matrices.

\section{A. Unimodular PU Matrices [24]}

Lemma 1 (PU Matrices [24]): Let $M$ and $P$ be two positive integers which are greater than one such that $P \mid M$. We consider $B H$ matrices $\mathbf{A}_{n}$ and $\mathbf{U}_{0}$ of size $P \times P$ and $M \times M$, respectively. We first take the following two matrices

$$
\begin{aligned}
\mathcal{U}_{n} & =\mathbf{I}_{\frac{M}{P}} \otimes \mathbf{A}_{n}, \\
\mathcal{D}(Z) & =\mathbf{I}_{\frac{M}{P}} \otimes \operatorname{diag}\left(1, Z^{-1}, \cdots, Z^{-(P-1)}\right),
\end{aligned}
$$

where $n \in\{1,2, \cdots, N\}$ for each positive integer $N$ and $\otimes$ is Kronecker product. Then, our recursive generator for unimodular PU matrix is given by

$$
\mathcal{G}_{n}(Z)=\mathcal{U}_{n} \cdot \mathcal{D}\left(Z^{D_{n}}\right) \cdot \mathbf{P}_{n} \cdot \mathcal{G}_{n-1}(Z) \cdot \mathbf{Q}_{n},
$$

where $\mathcal{G}_{0}(Z)=\mathbf{U}_{0}, D_{n}=P^{\pi(n-1)}, \pi$ is an arbitrary permutation of the numbers $\{0,1, \cdots, N-1\}$, and $\mathbf{P}_{n}, \mathbf{Q}_{n}$ are two arbitrary permutation matrices of equal size $M \times$ $M$. Then, $\mathcal{G}_{N}(Z)$ is a unimodular PU matrix of degree $P^{N}-1$.

The expanded form of the PU product from (6) is given by

$$
\mathcal{G}_{N}(Z)=\left\{\prod_{n=N}^{1}\left(\mathcal{U}_{n} \cdot \mathcal{D}\left(Z^{D_{n}}\right) \cdot \mathbf{P}_{n}\right)\right\} \cdot \mathbf{U}_{0} \cdot \mathbf{Q}_{0} .
$$

Example 2: Let $M=P=3$ and $N=2$ with $\pi=[1,0]$. Then, we have $D_{1}=M^{\pi(0)}=3$ and $D_{2}=M^{\pi(1)}=1$. Let $\mathbf{A}_{1}=\mathbf{A}_{2}=\mathbf{U}_{0}=\mathbf{F}_{3}$ and $\mathbf{P}_{1}=\mathbf{P}_{2}=\mathbf{Q}_{0}=\mathbf{I}_{3}$. According to (7), a $3 \times 3 \mathrm{PU}$ matrix $\mathcal{G}_{2}(Z)$ of sequence length $P^{N}=9$ is given by

$$
\mathcal{G}_{2}(Z)=\mathbf{F}_{3} \mathcal{D}(Z) \mathbf{F}_{3} \mathcal{D}\left(Z^{3}\right) \mathbf{F}_{3},
$$

where $\mathcal{D}(Z)=\operatorname{diag}\left(1, Z^{-1}, Z^{-2}\right)$.

\section{B. Proposed PU Construction of ZCZ Sequence Sets}

In this subsection, we will first propose a new construction of sets by using PU matrices from Lemma 1. Then, we will study the periodic correlation properties of the constructed sets.

Let $M$ and $P$ be two positive integers which are greater than one such that $P \mid M$. By using (7), a construction of $\mathrm{ZCZ}$ sequence sets is given by

$$
\begin{aligned}
\mathbf{x}(Z)= & \left\{\prod_{n=N}^{1}\left(\mathcal{U}_{n} \cdot \mathcal{D}\left(Z^{D_{n}}\right) \cdot \mathbf{P}_{n}\right)\right\} \cdot \mathbf{U}_{0} \cdot \mathbf{Q}_{0} \cdot \mathbf{D}^{P^{N}} \\
& \cdot \mathbf{F}_{M} \cdot \mathbf{D}^{M P^{N}} \cdot \mathbf{f}^{T},
\end{aligned}
$$

where $\mathbf{D}=\operatorname{diag}\left(1, Z^{-1}, \cdots, Z^{-(M-1)}\right)$ and $\mathbf{f}$ is any arbitrary row vector of a DFT matrix of size $M \times M$.

The above column vector $\mathbf{x}(Z)$ represents a set consisting of $M$ sequences with equal length $M^{2} P^{N}$. Due to the suitable delays and the product of unimodular complex numbers is unimodular complex number, the constructed sequences are unimodular sequences having equal length $M^{2} P^{N}$. Note that we can use any arbitrary PU matrix in (9) instead of just using the PU matrices from Lemma 1.

Remark 1: According to the previous works [23] and [24], it is easy to show that the constructed set $\mathbf{x}(Z)$ represents a CSS when we consider AACFs. Later, we will show that it is a ZCZ-CSS.

Let $\mathbf{x}(Z)=\left[x^{(0)}(Z), x^{(1)}(Z), \cdots, x^{(M-1)}(Z)\right]^{T}$ be the column vector constructed by (9). Then, the sequences $x^{(m)}(Z)$ can be expressed by

$$
x^{(m)}(Z)=\sum_{n=0}^{M-1} \sum_{k=0}^{M-1} a_{k} f_{n k} g_{m n}(Z) \cdot Z^{-(k M+n) P^{N}},
$$

where $g_{m n}(Z)$ is the $m n$-th sequence of the PU matrix given by (7) with $m, n \in\{0,1, \cdots, M-1\}$ and $\mathbf{f}=\left[a_{0}, a_{1}, \cdots, a_{M-1}\right]$ is any arbitrary row vector of $\mathbf{F}_{M}$. Note that each $a_{m}$ is an $M$-th root of unity. Equivalently, we can write (10) in time-domain as follows

$$
x^{(m)}[t]=c_{n k} \cdot g_{m n}\left[t-(k M+n) P^{N}\right],
$$

where $c_{n k}=a_{k} f_{n k}$ and $k \in\{0,1, \cdots, M-1\}$. Let us take $\mathbf{C}=\left[c_{n k}\right]$. Clearly, the matrix $\mathbf{C}$ has size $M \times M$. Later, we will study the periodic correlation properties of the row sequences of this matrix $\mathbf{C}$.

Since the values of each sequence $g_{m n}[l]$ outside the range $0 \leq l \leq P^{N}-1$ will be zeros, then, (11) becomes

$$
x^{(m)}[t]=x^{(m)}\left[(k M+n) P^{N}+l\right]=c_{n k} \cdot g_{m n}[l],
$$

where $t=(k M+n) P^{N}+l$. According to (12) and the product of unimodular complex numbers is unimodular complex number, one can observe that the generated sequences are unimodular sequences with length $M^{2} P^{N}$.

Remark 2: Based on our previous work [23] and [29], we can derive an efficient implementation of our proposed algorithm based on PU matrices for $\mathrm{ZCZ}$ sequence sets.

We are now ready to show that the column vector $\mathbf{x}(Z)$ represents a ZCZ sequence set.

Theorem 1: The column vector $\mathbf{x}(Z)$ given by (9) is a polyphase $\left(M, M^{2} P^{N},(M-1) P^{N}\right)$-ZCZ sequence set.

Proof: From (12), it is clear that the constructed sequences are polyphase sequences. To calculate the PCCF between any two constructed sequences, we first calculate PCCF between any two row sequences $\boldsymbol{c}_{n}$ and $\boldsymbol{c}_{n^{\prime}}$ of the matrix $\mathbf{C}=\left[c_{n k}\right]$ as follows

$$
\begin{aligned}
& \hat{R}_{\boldsymbol{c}_{n}, \boldsymbol{c}_{n^{\prime}}}[\tau]=\sum_{k=0}^{M-1} c_{n k} \cdot c_{n^{\prime}(k+\tau)_{M}}^{*} \\
& =\sum_{k=0}^{M-1} a_{k} \cdot f_{n k} \cdot a_{(k+\tau)_{M}}^{*} \cdot f_{n^{\prime}(k+\tau)_{M}}^{*} \\
& =\sum_{k=0}^{M-1} f_{(n+j) k} \cdot f_{\left(n^{\prime}+j\right)(k+\tau)_{M}}^{*}(0 \leq j \leq M-1) \\
& =\hat{R}_{f_{(n+j)}, f_{\left(n^{\prime}+j\right)}}[\tau]=\omega^{-(n+j) \tau} \cdot \delta\left(n-n^{\prime}\right) .
\end{aligned}
$$

To calculate PCCF between two sequences $x^{(m)}[t]$ and $x^{\left(m^{\prime}\right)}[t]$, we will use a similar logic used in [13]. So, the 
PCCF between two sequences $x^{(m)}[t]$ and $x^{\left(m^{\prime}\right)}[t]$ is given by

$$
\begin{aligned}
& \hat{R}_{\boldsymbol{x}^{(m), \boldsymbol{x}^{\left(m^{\prime}\right)}}}[\tau]=\sum_{t=0}^{M^{2} P^{N}-1} x^{(m)}[t] \cdot x^{\left(m^{\prime}\right) *}\left[(t+\tau)_{M^{2} P^{N}}\right] \\
& =\sum_{n=0}^{M-1} \sum_{k=0}^{M-1} \sum_{l=0}^{P^{N}-1} x^{(m)}\left[(k M+n) P^{N}+l\right] \\
& \cdot x^{\left(m^{\prime}\right) *}\left[\left(k+k^{\prime}+\Delta_{n}\right) M P^{N}+\left(n+n^{\prime}+\Delta_{l}\right) P^{N}+\left(l+l^{\prime}\right)\right. \\
& =\sum_{n=0}^{M-1}\left[\sum_{l=0}^{P^{N}-1} g_{m n}[l] \cdot g_{m^{\prime}\left(n+n^{\prime}+\Delta_{l}\right)}^{*}\left[l+l^{\prime}\right]\right. \\
& \left.\cdot \sum_{k=0}^{M-1} c_{n k} \cdot c_{\left(n+n^{\prime}+\Delta_{l}\right)\left(k+k^{\prime}+\Delta_{n}\right)}^{*}\right] \\
& =\sum_{n=0}^{M-1} R_{\boldsymbol{g}_{m n}, \boldsymbol{g}_{m^{\prime}\left(n+n^{\prime}\right)}}\left[l^{\prime}\right] \cdot \hat{R}_{\boldsymbol{c}_{n}, \boldsymbol{c}_{n+n^{\prime}}}\left[k^{\prime}+\Delta_{n}\right] \\
& +\left(1-\delta\left(l^{\prime}\right)\right) \cdot \sum_{n=0}^{M-1} R_{\boldsymbol{g}_{m n}, \boldsymbol{g}_{m^{\prime}\left(n+n^{\prime}+1\right)}}\left[l^{\prime}-P^{N}\right] \\
& \hat{R}_{\boldsymbol{c}_{n}, \boldsymbol{c}_{n+n^{\prime}+1}}\left[k^{\prime}+\Delta_{n}\right] \text {, } \\
& \hat{R}_{\boldsymbol{x}^{(m)}, \boldsymbol{x}^{\left(m^{\prime}\right)}}[\tau]=\sum_{n=0}^{M-1} R_{\boldsymbol{g}_{m n}, \boldsymbol{g}_{m^{\prime}\left(n+n^{\prime}\right)}}\left[l^{\prime}\right] \cdot \hat{R}_{\boldsymbol{c}_{n}, \boldsymbol{c}_{n+n^{\prime}}}\left[\Delta_{n}\right] \\
& =\sum_{n=0}^{M-1} R_{\boldsymbol{g}_{m n}, \boldsymbol{g}_{m^{\prime}\left(n+n^{\prime}\right)}}\left[l^{\prime}\right] \cdot \delta\left(n+n^{\prime}-n\right) \\
& =\sum_{n=0}^{M-1} R_{\boldsymbol{g}_{m n}, \boldsymbol{g}_{m^{\prime} n}}\left[l^{\prime}\right]=c \cdot \delta\left(m-m^{\prime}\right) \cdot \delta(\tau),
\end{aligned}
$$

where $\tau \leq(M-1) P^{N}$. Therefore, the IFW of $\mathbf{x}(Z)$ is $T=(M-1) P^{N}$. This completes the proof.

We observe that the periodic correlation properties of the matrix $\mathbf{C}$ play an important role in the expanded form of PU matrix in order to construct $\mathrm{ZCZ}$ sequence sets. The matrix $\mathbf{C}$ is designed by the entries of the last two unitary matrices in the expanded form (9). Therefore, the ZCZ property of the constructed sequences depends on the specific structure of the individual row sequences of the unitary matrices. On the other hand, in [23], it is shown that the use of equivalent forms of $B H$ matrices can significantly increase the number of constructed sequences. Consequently, we can construct a large class of ZCZ sequences based on PU matrices.

Remark 3: Note that $\mathbf{x}(Z)$ represents an $\left(M, M^{2} P^{N}\right)$ CSS when we consider AACFs and $\left(M, M^{2} P^{N},(M-\right.$ 1) $\left.P^{N}\right)$-ZCZ sequence set when we consider PCCFs. Therefore, the column vector $\mathbf{x}(Z)$ is a ZCZ-CSS of $M$ sequences with equal length $M^{2} P^{N}$.

The performance parameter $\eta$ of our proposed method is given by

$$
\eta=\frac{(M-1) P^{N}+1}{M P^{N}} \equiv 1-\frac{1}{M} .
$$

Consequently, $\eta$ is greater than $1 / 2$ as $M \geq 2$. According to (16), the performance parameter $\eta$ tends to 1 as $M$ increases. That is, the constructed sequence sets are asymptotic optimal ZCZ sequence sets. In other words, they are near-optimal ZCZ sequence sets.

We illustrate our proposed construction of $\mathrm{ZCZ}$ sequence sets based on PU matrices by the following example.

Example 3: Let $M=P=3$ and $N=2$. Let $\mathcal{G}_{2}(Z)=$ $\mathbf{F}_{3} \mathbf{D F}_{3} \mathbf{D}^{3} \mathbf{F}_{3}$ be a $3 \times 3$ PU matrix of sequence length 9 given in Example 2. Let $\mathbf{f}$ be the third row vector of $\mathbf{F}_{3}$. According to our proposed construction, the IFW is $T=(M-1) P^{N}=2 \cdot 3^{2}=18$ and sequence length is $M^{2} P^{N}=3^{2} \cdot 3^{2}=81$. Therefore, the constructed $(3,81,18)-\mathrm{ZCZ}$ sequence set $\mathbf{x}(Z)$ is given by

$$
\mathbf{x}(Z)=\mathbf{F}_{3} \mathbf{D} \mathbf{F}_{3} \mathbf{D}^{3} \mathbf{F}_{3} \mathbf{D}^{9} \mathbf{F}_{3} \mathbf{D}^{27} \mathbf{f}^{T},
$$

where $\mathbf{D}=\operatorname{diag}\left(1, Z^{-1}, Z^{-2}\right)$. We give this $(3,81,18)$ ZCZ sequence set in Table I, where only exponents of $\omega=e^{-2 \pi \sqrt{-1} / 3}$ are shown. The performance parameter is $\eta=\frac{(18+1) 3}{81}=0.704$.

The above example shows that we can generate ZCZ sequence sets with more flexible parameters (e.g., sequence lengths other than power of two) compared to the other techniques.

\section{Comparison with Previous Works}

In the literature, there are mainly three types of construction methods for ZCZ sequence sets. The first type is the direct construction method based on Boolean functions [8], [9]. The second type is based on perfect sequences [10], [11]. And the third type is based on MOCSSs [13], [14]. However, the sequence lengths and the set sizes for the constructions given in [8] and [9] are restricted to the powers of two only. In addition, for the constructions given in [10] and [11], the ZCZ parameters are limited by the existence of perfect sequences for the given number of phases. For instance, it is conjectured that no 4-phase perfect sequences of lengths other than $2,4,8$, and 16, exist [30]. Also, there does not exist a binary perfect sequence of length other than 4 [31]. Therefore, the method based on MOCSS is of strong interest since it has several benefits such as a wide variety of set sizes and sequence lengths compared to the other techniques.

Our proposed approach offers not only more choices in ZCZ parameter values compared to [8], [9], [10], and [11], but also provides a compact notation in treating matrix representations of multiple $\mathrm{ZCZ}$ sequence sets with performance parameter greater than $1 / 2$. For example, a $(3,81,18)$-ZCZ sequence set given in Example 3 cannot be obtained by the previous works [8], [9], [10], and [11]. In addition, our proposed method has an advantage over the previous works [13] and [14] about the fact that we can directly construct $\mathrm{ZCZ}$ sequence sets without constructing MOCSSs in advance.

\section{CONCLUSION AND FUTURE WORK}

In this paper, we have explored the application of $\mathrm{PU}$ matrices in the construction of ZCZ sequence sets. We have shown a connection between $\mathrm{ZCZ}$ sequence sets and PU matrices. More precisely, the periodic correlation 
Table I: A $(3,81,18)-Z C Z-C S S$ when $M=P=3$ and $N=2$

\begin{tabular}{|l|l|}
\hline $\mathbf{x}$ & 000012021000120210000201102222201210000120210111012210111120102000120210222120021 \\
& 012021000012102222012210111201210222012102222120021222120102111012102222201102000 \\
021000012021111201021222120210222201021111201102000201102111120021111201210111012
\end{tabular}

properties of the constructed sequences are entirely governed by the last two unitary matrices in the expanded product form of PU matrix. In addition, the number of constructed ZCZ sequences can be increased by using the equivalent forms of $\mathrm{BH}$ matrices. The proposed $\mathrm{ZCZ}$ sequence sets can be used in a QS-CDMA system to cope with asynchronous interference in multi-user transmission. An interesting future work is how to construct multiple ZCZ sequence sets with good inter-set cross correlation properties based on PU matrices.

\section{ACKNOWLEDGMENT}

S. Das is a visiting Ph.D. student with The University of Melbourne, Melbourne 3010, Australia, on academic leave from the Department of Mathematics, IIT Patna, India 801103. He would like to thank Dr. Srdjan Budišin at the RT-RK, Novi Sad, Serbia for the helpful discussions on PU matrices. The work of Z. Liu was supported in part by National Natural Science Foundation of China under Grant 61750110527, a Research Fund for International Young Scientists.

\section{REFERENCES}

[1] R. D. Gaudenzi, C. Elia, and R. Viola, "Bandlimited quasisynchronous CDMA: a novel satellite access technique for mobile and personal communication systems," IEEE J. Sel. Areas Commun., vol. 10, no. 2, pp. 328-343, Feb. 1992.

[2] H. Chen, S. Chu, and M. Guizani, "On next generation CDMA technologies: The real approach for perfect orthogonal code generation," IEEE Trans. Vehicular Technol., vol. 57, no. 5, pp. 2822 2833, Sep. 2008.

[3] S. Hu, Z. Liu, Y. L. Guan, C. Jin, Y. Huang, and J. Wu, "Training sequence design for efficient channel estimation in MIMO-FBMC systems," IEEE Access, vol. 5, pp. 4747-4758, 2017.

[4] H. Haderer, R. Feger, C. Pfeffer, and A. Stelzer, "Millimeter-wave phase-coded CW MIMO radar using zero- and low-correlationzone sequence sets," IEEE Transactions on Microwave Theory and Techniques, vol. 64, no. 12, pp. 4312-4323, Dec. 2016.

[5] R. Zhang, X. Cheng, M. Ma, and B. Jiao, "Interference-avoidance pilot design using ZCZ sequences for multi-cell MIMO-OFDM systems," in Proc. IEEE Global Communications Conference (GLOBECOM), Dec. 2012, pp. 5056-5061.

[6] X. H. Tang, P. Z. Fan, and S. Matsufuji, "Lower bounds on correlation of spreading sequence set with low or zero correlation zone," Electron. Lett., vol. 36, no. 6, pp. 551-552, Mar. 2000.

[7] Y. Li, C. Xu, and K. Liu, "Construction of mutually orthogonal zero correlation zone polyphase sequence sets," IEICE Tans. Fundamentals, vol. E94-A, no. 4, p. 1159-1164, 2011.

[8] Z. Liu, Y. L. Guan, and U. Parampalli, "A new construction of zero correlation zone sequences from generalized Reed-Muller codes," in Proc. IEEE Information Theory Workshop (ITW 2014), Nov. 2014, pp. 591-595.

[9] Y. Tang, C. Chen, and C. Chao, "A novel construction of zero correlation zone sequences based on Boolean functions," in Proc. IEEE 11th Int. Symp. Spread Spectrum Techniques and Applications, Oct. 2010, pp. 198-203.

[10] H. Torii, M. Nakamura, and N. Suehiro, "A new class of zerocorrelation zone sequences," IEEE Trans. Inf. Theory, vol. 50, no. 3 , pp. 559-565, Mar. 2004.
[11] Z. Zhou, X. Tang, and G. Gong, "A new class of sequences with zero or low correlation zone based on interleaving technique," IEEE Trans. Inf. Theory, vol. 54, no. 9, pp. 4267-4273, Sep. 2008.

[12] Z. Zhou, X. Tang, and D. Peng, "New optimal quadriphase zero correlation zone sequence sets with mismatched filtering," IEEE Signal Process. Lett., vol. 16, no. 7, pp. 636-639, Jul. 2009.

[13] C. Han, T. Hashimoto, and N. Suehiro, "A new construction method of zero-correlation zone sequences based on complete complementary codes," IEICE Trans. Fundamentals., vol. E91-A, no. 12 , pp. 3698-3702, 2008.

[14] A. Rathinakumar and A. K. Chaturvedi, "A new framework for constructing mutually orthogonal complementary sets and ZCZ sequences," IEEE Trans. Inf. Theory, vol. 52, no. 8, pp. 38173826, Aug. 2006.

[15] C. C. Tseng and C. L. Liu, "Complementary sets of sequences," IEEE Trans. Inf. Theory, vol. 18, no. 5, pp. 644-652, Sep. 1972.

[16] S. Budišin, "New complementary pairs of sequences," Electron. Lett., vol. 26, no. 13, pp. 881-883, Jun. 1990.

[17] J. A. Davis and J. Jedwab, "Peak-to-mean power control in OFDM, Golay complementary sequences, and Reed-Muller codes," IEEE Trans. Inf. Theory, vol. 45, no. 7, pp. 2397-2417, Nov. 1999.

[18] N. Suehiro and M. Hatori, "N-shift cross-orthogonal sequences," IEEE Trans. Inf. Theory, vol. 34, no. 1, pp. 143-146, Jan. 1988.

[19] S. Z. Budišin and P. Spasojević, "Paraunitary generation/correlation of QAM complementary sequence pairs," in Proc. Cryptography and Communications 6.1, Oct. 2014, pp. 59-102.

[20] P. P. Vaidyanathan, Multi-rate systems and filter banks. Prentice Hall, 1993.

[21] Z. Wang, G. Wu, and D. Ma, "A new method to construct Golay complementary set by paraunitary matrices and Hadamard matrices," in Proc. 9th International Conference on Sequences and Their Applications (SETA-2016), Sep. 2016, pp. 1-12.

[22] S. Z. Budišin and P. Spasojević, "Paraunitary-based Boolean generator for QAM complementary sequences of length $2^{K}$," IEEE Trans. Inf. Theory, vol. 64, no. 8, pp. 5938-5956, Aug. 2018.

[23] S. Das, S. Budišin, S. Majhi, Z. Liu, and Y. L. Guan, "A multiplierfree generator for polyphase complete complementary codes," IEEE Trans. Signal Process., vol. 66, no. 5, pp. 1184-1196, Nov. 2017.

[24] S. Das, S. Majhi, and Z. Liu, "A novel class of complete complementary codes and their applications for APU matrices," IEEE Signal Process. Lett., vol. 25, no. 9, pp. 1300-1304, Jul. 2018.

[25] S. Das, S. Majhi, S. Budišin, Z. Liu, and Y. L. Guan, "A novel multiplier-free generator for complete complementary codes," in Proc. 23rd Asia-Pacific Conference on Communications (APCC), Dec. 2017, pp. 1-5.

[26] D. Ma, S. Budišin, Z. Wang, and G. Gong, "A new generalized paraunitary generator for complementary sets and complete complementary codes of size $2^{m}$," IEEE Signal Process. Lett., vol. 26, no. 1, pp. 4-8, Oct. 2018.

[27] S. Das, S. Majhi, S. Budišin, and Z. Liu, "A new construction framework for polyphase complete complementary codes with various lengths," IEEE Trans. Signal Process., vol. 67, no. 10, pp. 2639-2648, May 2019.

[28] A. T. Butson, "Generalized Hadamard matrices," in Proc. Am. Math. Soc. 13, 1962, pp. 894-898.

[29] S. Das, S. Majhi, and P. Sarkar, "An improved multiplier-free generator for polyphase complete complementary codes," in Proc. 10th International Conference on Sequences and Their Applications (SETA-2018), Oct. 2018, pp. 1-12.

[30] W. H. Mow, "A unified construction of perfect polyphase sequences,” in Proc. IEEE ISIT, Sep. 1995, pp. 459-.

[31] B. Schmidt, "Cyclotomic integers and finite geometry," Jr. Am. Math. Society, vol. 12, no. 4, pp. 929-952, 1999. 